cases bearing a more or less close resemblance to it-cases of P.U.O. which suggested pneumonia and were not pneumonia or broncho-pneumonia, but something else. One of us (W. P.) has for months past directed the attention of medical officers to the fact that in cases of P.U.O., without any focal signs or symptoms, careful examination of the bases sometimes reveals the presence of slight, apparently deep-seated, change in one or other lung, and drawn attention to the peculiar fine sticky râle in deep inspiration which was such a marked feature in the case we have just described-a râle, by the way, which has nothing in common with the fine râles of œrlema, but suggests the passage of air under pressure into collapsed bronchioles and alveoli.

We do not wish to imply that there is a cut-and-dried category of P.U.O. which conforms strictly to the case we have just described. If the occurrence of localised lesions of the deeper bronchi is a reality, it would follow almost of necessity that their effects on the lungs would vary from case to case according to their situation and extent.

Is it not possible that the featureless aspect of so many cases diagnosed as P.U.O. may be just because they are the clinical expression of smouldering bacterial infections which -for reasons it would be profitless to speculate upon herehave failed to become fully alight (active) and thereby more individualised and recognisable in their outward expression? Had the infection in our patient been more active and shown a tendency to spread we should without doubt have had on our hands a case of pneumonia or broncho-pneumonia instead of one which, under existing conditions, we can only label P.U.O.

\section{THE LOCALISATION AND REMOVAL OF FOREIGN BODIES BY THE COMBINED METHOD OF RADIOSCOPY AND OPERATION,}

WITH SUGGESTIONS FOR THE STANDARDISATION OF METHODS, BY ROBERT KNOX, M.D. EDIN.,

CAPTAIN, R.A.M.C. (T.). ; CONSULTING RADIOLOGIST, GREAT NORTHERA OENTRAL HOSPITAL; AND RADIOLOGIST, KING'S COLLEGE HOSPITAL.

IN view of the large number of papers already published dealing with the localisation of foreign bodies any further publication on the subject might at first sight appear to be superfluous, and it may be admitted that this consideration has for long restrained the writer from publishing anything on the subject. Many of the papers detail admirably the technique of localisation. Most of them are more or less practical and useful, but a consideration of the literature of localisation forces the conclusion that there is still something lacking, though exactly what it is is very difficult to define.

\section{Consideration of Present Position.}

The existence of so many methods clearly indicates the need for standardisation, not only of methods of localisation, but also of the apparatus by means of which the necessary evidence is obtained. Such standardisation of methods of localisation and apparatus used would soon lead to a uniformity of results. The adoption of one good method universally for the localisation and removal of foreign bodies would be an important step towards this end.

From a considerable experience in localisation of foreign bodies, the interpretation of the results, and the subsequent operative measures for their removal the writer is compelled to admit that in many instances the information provided by the radiologist does not convey in an intelligible manner the necessary information to the surgeon. It is either a bald statement of facts and measurements, or it is an exhibition of negatives from which the surgeon is expected to deduce the information. A pair of flat negatives in two positions may give the necessary information in a number of cases, but in the majority they fail to do so. Stereoscopic plates, if intelligently interpreted, give all the information required, but many surgeons are unable to appreciate the information when so conveyed.

Experience in the operating-room will soon convince the radiologist that however much he may pride himself on the accuracy of his localisation (and in the majority of cases where accurate technique is employed there can be no question on this point) he has totally failed to give the surgeon a mental picture of the exact location of the foreign body and its relationship to anatomical landmarks in its vicinity. That is the crux of the whole matter, once a dissection has been begun the marks on the skin become practically vaiueless. The subsequent procedure on the part of the surgeon in difficult cases ends in a blind probing in an endeavour to discover the foreign body; hours may be wasted in some cases, and in the end failure is the result.

The endeavour of the radiologist must therefore be, if localisation alone is relied upon, to give the relationship of the foreign body to well-known landmarks as well as its depth from the surface. This can be rendered more valuable. by the adoption of what might be described as the graphic method of demonstration by means of carefully drawn diagrams on which the exact location of the foreign body is marked, and its depth given from the skin surface. This method will be found extremely useful in a number of cases, but in others it will be found that there is something wanting. A further step towards accuracy in localisation and success in removal would be the collaboration of the surgeon and the radiologist in the operating-room. This will be found to be the most satisfactory of all.

The object of the writer will therefore be to refer (1) to the best known methods of localisation in order that the surgeon may see the matter from the radiological point of view; (2) the graphic interpretation of the result to the surgeon ; and (3) the combined method of radiography and surgery in the operating-room.

It will be necessary, therefore, to deal with the matter under three distinct headings, namely :-

1. The localisation of foreign bodies.

2. The interpretation of the results (graphically).

3. The methods which may be employed in the operating theatre, by means of $\mathrm{X}$ ray apparatus for localisation and as an aid to removal at the time of operation. The latter method has been described as that of intermittent control by radioscopy at the time of operation

This method has recently been described by two French radiologists, MM. Ombredanne and A. Ledoux-Lebard, in a paper published in Journal de Radinlogie et l' Eleotrologie (vol. ii., March, April, 1916), but it is only fair to radiologists in general to point out that the method has been in use in this country for years and has become so generally adopted that it is a matter for surprise that so much attention should be called to it now.

Many years ago the writer employed the method at the Great Northern Central Hospital in collaboration with $\mathrm{Mr}$. Edmunds for the removal of needles in the limbs of patients. Since the outbreak of war the method has been revived at the 4th London General Hospital in collaboration with colleagues there, and a number of cases have been operated on with success; in these cases remoral had been in some instances attempted without success, the usual methods of localisation having been employed.

In view of these facts it has been considered expedient to revise the whole method of localisation and combined operation, in the hope that by so doing it will be possible to call attention to the subject and lead to a closer coöperation of the radiologist and surgeon under what may be described as the ideal method of localisation and removal of foreign bodies.

Precautions to be Observed.

Before entering into detailed descriptions there are several important matters to which attention must be called. The first is (a) the danger that undoubtedly exists for the operators when a large amount of screening is done; $(b)$ the methods of preventing these davgers; and $(c)$ the undoubted danger of $X$ ray burning of the patient if too lengthy examinations are carried out.

Screening should only be employed for the shortest possible time, consequently the conditions under which it is conducted must be the best obtainable.

The chief of these is to provide for absolute darkness when the $X$ ray tube is in action. This can be done by making the operating room light-proof and by covering all valve tubes; the $\mathrm{X}$ ray bulb can be completely enclosed in the ray-proof box. The operating room should have a double supply of lights. Powerful ruby or blue lights may be emploved, by means of which the surgeon can carry out the necessary operative measures; if these are used the strain on the retina is minimised, so that when the $X$ rays are in operation the observer can rapidly examine and locate 
the foreign body. This lewds to a considerable shortening of the time necessary to carry out the examination. Ordinary light can be used if absolutely necessary for operative work. The radiologist should then use coloured spectacles or should have a hood fitted over the head to exclude the white light.

The $X$ rav bulb should be kept at a suitable degree of penetration to enable the observer quickly to make out the position of the foreign body. The bulb must be efficiently protected by thick sheets of lead incorporated in the wood of the box which containg the tube; the smallest possible aperture in the diaphragm should be employed when examining for the foreign body. The protection of the patient is secured if a plate of alumininm 2 or $3 \mathrm{~mm}$. thick is placed between the $X$ ray bulb and the patient to minimise the effect on the skin, and the duration of the exposure kept well within what would produce an erytherna of the skin.

Whatever precautions are employed the possibility of damage to the patient's skin and to the operator's must never be lost sight of, consequently the shortest possible time for examination is the best of all precautions. The operator carries out a preliminary dissection, acting on a previously made localisation. When necessary he asks for a screen examination, and so on until the foreign body is discovered and removed.

\section{Methods of Localisation.}

(a) Plate method: (1) With the $\mathrm{X}$ ray tube above the patient; (2) with the $X$ ray tube below the patient; (3) stereoscopic localisation: (a) tube above the patient; (b) tube below the patient.

(b) Soreen methods of lncalisation - These necessarily consist of examination with the patient on the $X$ ray couch, tube below the couch, the fluorescent screen above the part to be examined.

It may be assumed that the principles of localisation of foreign bodies by means of $X$ rays are generally uuderstood; for our present purpose it will suffice to deal briefly with the methods generally employed and point out the advantages each possesses.

Plate methods. - On considering the plate methods we find that there are two positions of the tube: $(a)$ above the subject, the plate being placed below; (b) the tube below the subject-i.e., placed under the couch or table upon which the patient lies. The platse method possesses several advantages over the screen method as follows:-

The chief advantage is the production of a permanent record of the foreign body, itg relationship to bone, and the existence of an injury to the bone or soft parts in its vicinity. This is in itself an important argument in favoux of this method becoming a standard one. Another and by no means an unimportant advantage is the fact that it does not lead to the exposure of the operator to the injurious action of the rays. When the tube is placed below the table the plate method may be combined with the screen method when a record of the condition observed is required.

The stereoscopic method is undoubtedly one of the most useful, as by its use information as to the exact position of foreign body and its relationship to anatomical landmarks in the vicinity can be obtained. It is possible to demonstrate the exact position of a foreign body when the two plates are examined in the stereoscope. This method will be found to be a most valuable aid to the determination of position in difficult cases, but there is very little likelihood of it ever being adopted as a standard method, because of the heavier expense and time occupied in producing the plates. The stereoscopic method may be empioyed with the tube either above or below the couch.

Screen methods.-These are very numerous-some are ex ceedingly ingenious-all are based on the same genera principle. The localisation can be carried out entirely by the screen, or plates may be employed for purposes of corroboration. The distance from the anticathode of the tube and the surface of the plate must be known-when possible it is well to maintain a fixed distance between these two points, then a table of measurements calculated for that distance can be prepared which gives the depth of the foreign body for a particular displacement of the shadow. The tube displace ment should also be a constant quantity. When the plate is employed two exposures on une plate will be sufficient for practical purposes.

Aids to the Interpretation of the Hxact Position of Foreign Bodies.

When radiographic measures have been employed it is possible, after a simple procedure of calculation and measurement, to state that the foreign body occupies a definite position in relation to the marks which have been placed upon the skin surface, and to note its relationship to the anatomical landmarks in the ricinity. Stereoscopic olates

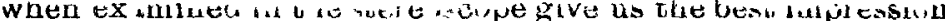
of the position of the foreign body and its relations to anatoms cal landmarks and to marks placed upon the skiu surface It is most valuable because $1 \mathrm{t}$ is the only mi ans we ponsess of visualising the parts and conveying to the sureson an exict appreciation of the location and of ten the diffoulties likely to complicate an operation for its removal. A kno vledge of anatomy enables a mental pioture of the powition of the foreign body to be formed which will be usteful when an operation is carried out for its removal. This is the most satisfactory method of interpretation we possess because it enables us to reach the foreign body independently of the skin markings, which latter are frequently of no value after the preliminary incision has been made. A thorough knowledge of anatomy is, therefore, essential on the part of both the surgeon and the radiologist, and enables the conveying of the findings of the radiologist to the surgeon to become an easy and reliable procedure.

A series of radiographic charts would be found useful in reporting the results of localisation, and several valuable aids exist towards the compilation of such charts. Of these, attention must be called to a paper by Dr. J. Metcalfe and Dr. E. M. Keys-Wells, published in THE LANcex, May 27th, 1916. This gives in outline a table of measurements, made upon the cadaver, of the prominent bony parts in the body. These tables might quite readily become the basis of a series of standard measurements.

Professor Symington has produced a valuable work on Sectional Anatomy which will be found to be of the greatest possible value to the radiologist and surgeon in their efforts to localise and remove foreign bodies-and it should facilitate the work of the radiologist in conveying his results in an intelligible fashion to the surgeon. Captain P. T. Crymble has called attention to the value of sectional anatomy in this relationship in a paper published in the British Journal of Surgery, vol. iv., Nov. 14th, 1917.

A careful stuay of the admirable stereo-photographs in the "Edinburgh Stereoscopic Atlas of Anatomy" will also repay the radiologist whose knowledge of practical anatomy has become rusty from lack of practice and the lapse of years. When stereoscopic radiographs have been taken a comparison of these with the corresponding anatomical part photographed stereoscopically should be of use in definitely localising the foreign body.

Thus, careful comparison of the radiographic findings, anatomical illustrations, and stereoscopic photographs should enable us to determine the exact position of the foreign body and its relations to anatomical landmarks. It is still necesaary to illustrate the findings graphically, since the radiologist may not always be in intimate touch with the surgeon. For this purpose I am having prepared a series of drawings from anatomical and radiographic studies which may be regarded as standards for the purpose of issuing a report which will be intelligible and indicate the position of the foreign body in its relationship to anatomical structures in its vicinity.

Coinbined Localisation and Operative Measures.

of all the methods so far employed whereby the surgeon and the radiologist collaborate, undoubtedly the most satisfactory is that of the combined work in the operatingroom. When the pressure of work is very great the employment of this method is most economical in several respects. It obviates the use of a large number of photographic plates and the time necessary for their production. This is in itself an important factor in favour of the use of the combined method. It saves the time of the radiologist and surgeon in their joint consideration of the finished plates. But the chief advantage is the great saving of time in the operatingroom. By this combined method it should be possible quickly to localise and remove any foreign body which is surgically accessible. After the preliminary localising measures have been taken the surgeon is in a position to proceed at once with the operation. Should he fail to tind the foreign body quickly it is only necessary to darken the room, examine for the pocition and, if necessary, re-localise the foreign body. In difficult cases the instrument may even be guided to the foreign body. The saving of time which follows upnn the combined method may be in a number of cases very considerable. Instead of one or even two hours spent in exploring for a foreign body situared in a difficult position the whole operation may be reduced to a quarter or half an hour. When a considerable amount 
of work has to be got through this is a most important matter.

Assuming, therefore, that the combined method is the best of all for the localisation and removal, what are the essentials for the efficient execution of the combined effort ? The chief of these are :

(1) Accuracy of localisation. This may be secured by adopting the most perfect mechanical apparatus available.

(2) The next and not the least important condition is that the method must be carried out with due regard to the protection of : (a) the operators and assistants, and $(b)$ the patient.

(3) Another important matter is that the operation must be conducted under ideal surgical conditions. 'The operating room must therefore be a perfect one with all surgical acsessories, and at the same time be capable of being converted into an absolutely dark room ; from the radiographic point of view this is most imporsant. Given a perfectly darkened room then the radioscopic measures may be carried out with expedition and success.

It will therefore be necessary to consider these various points in order and at considerable length, if we are to arrive at a satisfactory explanation of this most useful method.

For the employment of the screen method of localisation it is generally necessary to examine the patient while he is lying upon a table or $\mathrm{X}$ ray couch. Other positions are only possible when the patient is not under an anæs shetic, so for practical purposes the description must be confined to the examination on the operating couch. The arrangement of the $X$ ray tube and the localising apparatus up to the present is largely a matter of choice upon the part of the radiologist ; so long as accuracy is secured it matters little what method is employed, but since in this article the intention is to indicate the possibility of standardisation of method, apparatus, and the recording of results, what in the writer's opinion, at least, is the best method, will be described.

The description may be limited to several well-known pieces of apparatius: (1) the Mackenzie Davidson localising table; (2) the Bruce director couch (with modifications); (3) Baese's localiser and explorer; and (4) a suggestion for a new table constructed on similar lines to the first two.

\section{The Localising Couch.}

Nearly all of the practical methods of localisation now in use are bised on the work of Mackenzie Davidson. The most commonly used method is to place the patient upon a couch, the tube under the table. the fluorescent screen and localising device above the patient. This is the position which commends itself for operative measures, so it might reasonarly be suggested as the standar.l position for localising and operating. All cases, however, do not require operation. It would still be a rational measure to examine all cases in the position most suitable for operation.

The difficulty in this method up till quite lately has been to get the pirt examined at a fixed distance from the tube, a most important point in standardisation. This has led to the production of a number of tables calculated for various tube distances. Mickenzie Davidson has overcome this by having a fixed distance between tre tube and the screen-by mechanical adjustments the surface of the screen is arranged to make contact with the skin surfacethe tube moves vertically up and down in the tube box. Furthermore, he has done away with the need for tables by adding an ingenious device which records the displacement of the object when the tube is moved and automatically registers the depth of the foreign body.

Baese in his localiser and explorer has also adopted the fixed distance between the tube and the screen. Davidson ascertains the depth by a displacement of the tube in one direction. Baese measures the depth by a displacement of a graduated scale attached to the fluorescent screen. Either of these methols might be adopted. For practical purposes in this country Davidson's will be found to be the more readily adapted.

For operative purposes the Mackenzie Davidson apparatus can readily be arranged if a surgical table on four legs and having a stout aluminium top is provided.

The tube with its protected holder and localising device, which might for convenience be referred to as the localising unit, can be fitted up independently of the table; then it is only necessary to arrange the table with the patient over the tube box, the part is arranged for localisation, and table, patient, and part so arranged fixed by clamps. The localisation s quickly carried out, the skin is marked. The radiologist now stands aside, the localising unit is removed, and the surgeon proceeds with the operation. He has a spot marked on the skin and has been told the depth. Should he not quickly find the foreign body the localising unit is again brought into position and the foreign body re-localised. If difficulty still persists the surgeon can be directed to the foreign body by screening while operating. This latter procedure should be used as little as possible. Over-exposure to $\mathrm{X}$ rays will sooner or later lead to dermatitis. This to a practising surgeon would be little short of a calamity. Rubber gloves worn by the surgeon offer no protection against $X$ rays, although some surgeons have asserted that they do. All operators using $X$ rays while operating must keep the hands and arms well away from the localising beam of rays.

The tube must be thoroughly shut in by lead or lead-glass. Mackenzie Davidson's latest table is admirably furnished with a large well-lined tube box which cuts off all but the rays emitted from the diaphragm aperture.

The whole of the "localising unit" could be made of metal, and would then have all the precision, if well constructed, of a micrometer scale; this would ensure accuracy in estimating the position and depth of a foreign body. Most of the apparatus in present use lacks the final finish in construction which is so conspicuous in properly constructed mechanical apparatus. The measuring instruments on a localising couch should be as precise and accurate as the instruments used on a gun. Accuracy in estimation of distance in both means successful execution of purpose. The $X$ ray table must be constructed to admit of it being readily arranged over the "localising unit." It also can be made of metal, but the top must be of some material which is not too opaque to the $\mathrm{X}$ rays. Aluminium of $2 \mathrm{~mm}$. thick would serve admirably for this purpose.

Dr. Ironside Bruce has introduced a couch which might readily be adapted to carry out the preliminary localisation and the subsequent procedure for the removal of the foreign body. ${ }^{1}$ A director is attached to the arm which in the preliminary observations carries the fluorescent screen; this indicates the direction which the surgeon must follow to reach the foreign body. By substituting a larger fluorescent screen than that used by Bruce and making a few alterations in the details this would be an admirable combined radioscopic and operating table.

Signor Baese has introduced a localiser and explorer which is most ingenious in its application, and with a few modifications might readily be adopted as a localising unit for use in the operating theatre. This localis $\rightarrow r$ has been described by Professor J. Bergonié in the Archives d'Electricité Médicale et de Physio-Thérapie, February, 1917. Baese has rejected generalised methods and all calculations; in his method the centre of rotation is movable in the direction of the " normal ray" or "in a plane parallel to it," and thus the axis of rotation is made to pass through the body to be localised. The intersection is determined by the simple criterion-i.e., the mobility or immobility of the shadow when the axis of rotation intersects the foreign body, and when the position of the centre of rotation is known the position of the foreign body is established.

\section{Modification of a Localising Couch to Operative Requirements.}

Since any part of a patient's body must be readily adjusted in the path of the central rays of an $X$ ray bulb-i.e., between the focus point of the tube and the central point where the rays emitted from it impinge upon the fluorescent screen-it is essential that the table should have rapid mechanical movements. Some modification of the existing tables appears to be indicated. The suggestion is that the tube box, with its fittings and localising attachments, should be made separately from the table. The Baese localiser and explorer is admirably adapted to this purpose, it is complete in itself, can readily be removed from the table, and is quickly readjusted; it is, therefore, an excellent "localising unit." When a unit of this type is used the operating table may be of the ordinary type so long as it is possible to place the $\mathrm{X}$ ray tube box under it.

When the localising unit is fixed the table used for the operation should be capable of movement in two directions. This can readily be attained if it runs on rails allowing of movement in two directions; immediately the part to be examined is arranged in the path of the central 
beam the table is clamped in position. The table may consist of a metal frame, the top should be of aluminium sufficiently thick to ensure rigidity; this aluminium top serves a double purpose, it protects the patient's skin from the action of the softer $X$ rays by absorbing them and it is readily sterilised. $^{2}$

The essential part of the equipment is the localising device and the $\mathrm{X}$ ray bulb. Undoubtedly one of the best at present in use is that recently introduced by Mackenzie Davidson. A valuable addition to the table would be to fit it with a second tube box so placed that a lateral view could readily be obtained.

This might be achieved in two ways: (1) By having attached to the tube box a swing-over ratchet which would carry the tube box to an exact right angle from the original position; (2) a second tube box, accurately centred, could be arranged in position, so that, by using a change-over footswitch, the current could quickly be changed from one tube to the other. An arrangement of this kind, in whatever way obtained, would be extremely helpful in difficult cases.

\section{Other Points.}

The assumption has been made that localisation prior to operation will become a routine measure in all hospitals.

The radiologist will, however, examine many cases which do not proceed to operation. It will therefore be necessary for him to report on the exact position of the foreign body in order that the surgeon may be able to decide upon the appropriate treatment. The localisation should be carried out in precisely the same way as when operation is contemplated.

The report on the case should be amplified in order to convey, if possible, an idea of the position of the foreign body in relation to anatomical landmarks. A careful consideration of the negatives, depth measurement, Metcalfe's and Keys-Wells's tables, Symington's Atlas (Sectional), with in difficult cases stereoscopic plates, shuuld enable the radiologist graphically to demonstrate its position. For this purpose, as already mentioned, I am having a series of diagrams prepared. By the aid of charts of this kind the work of the radiologist is rendered intelligible to those not familiar with radiographic technique. These diagrams could readily become a unit in a standardised scheme.

The close collaboration of the surgeon and the radiologist is essential in a scheme of this kind. Surgeons as well as radiologists should familiarise themselves with the necessary principles of localisation-particularly stereoscopic, which is an invaluable aid to the appreciation of position in a difficult case. A few hours spent in the $X$ ray department by all operating surgeons would be a most profitable step towards perfection in operating for the removal of foreign bodies.

When difficulties in removal arise it is not always the radiologist who is at fault, frequently the surgeon is hopelessly wrong in his appreciation of the exact position of the foreign body. Occasionally anatomical considerations may render it impossible to remove a foreign body; in such cases if the surgeon has received accurate information as to position he should have realised the difficulty and have refrained from operating. Several instances of this kind have occurred where, when the difficulty of removal was great, the surgeon has wisely refused to proceed with the - operation for removal. Surgeons as well as radiologists vary in their power to appreciate the difficulties of a localisation.

The last stage in the standardisation of methods would be to arrange for the instruction of all radiologists appointed to the charge of $X$ ray departments at a convenient centre prior to going on service. This could be done at a school or hospital where a department would be fitted up on standard lines with all the necessary apparatus for accurate work. A complete collection of plates illustrating typical cases would be useful for purposes of demonstration. At such a school surgeons would be welcomed and demonstrations given on difficult points.

Conolusions.

If a standard method of localisation is to be developed it appears that the fluoroscopic offers the strongest claims to its adoption for the following reasons :-

1. It is convenient for the radiologist and essential from the operative point of view, and when combined with the natter it is the method which will be most successful.
2. It is most economical from both points of view-i.e., it saves the time of the operators and the cost of plates.

3. When combined with scientifically constructed localising apparatus it is mathematically accurate.

4. It enables the operators to re-localise and so correct any error which may have occurred.

5. It may be used as a guide to the surgeon. It could be improved upon in this respect if stereoscopic fluoroscopy became a practical entity. That this is possible there is no shadow of doubt. The method could with a little experimental work become thoroughly practical. That this is so is proved by work being done in France and America.

Should the fluoroscopic method become general it must be insisted upon that all protective measures must be employed. Operators must keep out of the way of the active radiations if disaster is to be avoided. Surgeons must realise the importance of not exposing themselves unnecessarily to the rays; consequently it should only be used for very short periods of time as a controlling agent. Apparatus must be carefully examined to ensure that all the protective measures are really safe.

In difficult cases the plate method, and particularly the stereoscopic, will be found indispensable-the object of its use being to present to the surgeon a picture of the exact position of the foreign body. The plate method cannot be dispensed with because there are occasions when the foreign body cannot be satisfactorily seen with the screen method; in these cases the plates will give more helpful aid than the screen. Foreign bodies in the eye and orbit, skull, spine, and pelvis should always be localised by the plate method prior to operation. The screen method may in these cases be used as an aid at the operation. The telephone probe will be found most useful in dealing with difficult cases. ${ }^{2}$

The adoption of standard methods could not be carried out simultaneously throughout the numerous military hospitals now on active service. It is obvious that the process of standardisation must be a gradual one, but if decided upon it would be possible to fit up all new hospitals with standard apparatus. The initial cost of fitting all hospitals with standard outfits and $\mathrm{X}$ ray operating-theatres would be a heavy item from the financial point of view alone. It is, however, quite unnecessary to do this; indeed, it would be wasteful. Several hospitals at the base and in England could be equipped for the work, and all difficult or doubtful cases referred to these hospitals for investigation and operation. In this way several surgical experts could undertake all the operative work for the removal of foreign bodies. They would have the advantage of expert radiologists working with reliable apparatus to help them in deciding upon operation, and in the operating. theatre when difficult cases were undertaken a large amount of most useful work could be systematically dealt with.

\section{THE TREATMENT OF STAPHYLOCOCCAL INFECTIONS BY STANNOXYL : FURUNCULOSIS AND ACNE.}

\section{(Method of GRÉGOIRE and FROUIN.)}

\section{By ARTHUR COMPTON, B.A., M B R.U.I., D.Sc. N.U.I.,} CAPTAIN, R.A.M.c. (TEMPORARY); OFFIOER IN CHARgE MILITARY BACTERIOLOGICAL LABOBATORY, DOHSET DISTRICT; FORMGRLY Research assistant, INSTITUT Pasteur, Paris.

Frouin, ${ }^{1}$ in pursuing his interesting researches on the action in vitro of metals, particularly the rare metals, on different bacteria, has recently been led to investigate the influence of tin and its derivatives on the growth of staphylococci. With Grégoire ${ }^{2}$ he has established that this element, its chloride or its oxide, when added to ordinary bouillon culture-medium, strongly inhibits the growth under anaerobic conditions of staphylococci; while under aerobic conditions the growth of staphylococci is not hindered, but the virulence of the microbe is diminished. That fact, coupled with the observation coming from the district of Beance that furunculosis is an unknown affection among tin-work rs of the region, formed for Frouin and Grégoire the startingpoint for testing in vivo the therapeutic value of tin in staphylococcal infections. They found that intravenous

2 The Lancet, 1915, 1., 217. 